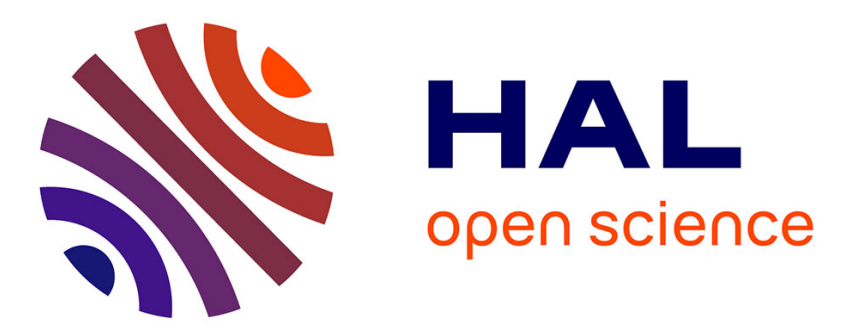

\title{
Independent Component Analysis Based on First-Order Statistics
}

\author{
Vicente Zarzoso, Rubén Martín-Clemente, Susana Hornillo-Mellado
}

\section{To cite this version:}

Vicente Zarzoso, Rubén Martín-Clemente, Susana Hornillo-Mellado. Independent Component Analysis Based on First-Order Statistics. Signal Processing, 2012, 92 (8), pp.1779-1784. hal-00848550

\section{HAL Id: hal-00848550 \\ https://hal.science/hal-00848550}

Submitted on 26 Jul 2013

HAL is a multi-disciplinary open access archive for the deposit and dissemination of scientific research documents, whether they are published or not. The documents may come from teaching and research institutions in France or abroad, or from public or private research centers.
L'archive ouverte pluridisciplinaire HAL, est destinée au dépôt et à la diffusion de documents scientifiques de niveau recherche, publiés ou non, émanant des établissements d'enseignement et de recherche français ou étrangers, des laboratoires publics ou privés. 


\title{
Independent Component Analysis Based on First-Order Statistics
}

\author{
V. Zarzoso ${ }^{\mathrm{a}, 1, *}$, R. Martín-Clemente ${ }^{\mathrm{b}, 2}$, S. Hornillo-Mellado $^{\mathrm{b}, 2}$ \\ ${ }^{a}$ I3S Laboratory, University of Nice Sophia Antipolis, CNRS, 2000 route des Lucioles, BP 121, 06903 Sophia Antipolis \\ Cedex, France \\ ${ }^{b}$ Departamento de Teoría de la Señal y Comunicaciones, University of Seville, Escuela Superior de Ingenieros, Avda. de \\ los Descubrimientos, s/n, 41092 Seville, Spain
}

\begin{abstract}
This communication puts forward a novel method for independent source extraction in instantaneous linear mixtures. The method is based on the conditional mean of the whitened observations and requires some prior knowledge of the positive support of the desired source. A theoretical performance analysis yields the closed-form expression of the asymptotic interference-to-signal ratio (ISR) achieved by this technique. The analysis includes the effects of inaccuracies in the estimation of the positive support of the desired source in single-step and iterative implementations of the algorithm. Numerical experiments validate the fitness of the asymptotic approximations. As it is based on first-order statistics, the method is extremely cost-effective, which makes it an attractive alternative to second- and higher-order statistical techniques in power-limited scenarios.
\end{abstract}

Keywords: Independent component analysis, conditional statistics, performance analysis.

\section{Introduction}

Independent component analysis (ICA) has received considerable attention over the last decades, mainly due to its wide variety of application domains. In particular, ICA is the key tool for blind source separation in instantaneous linear mixtures when the sources are statistically independent, an assumption quite plausible in many scenarios. Seminal work on ICA focused on the direct exploitation of statistical independence through higher-order statistics (HOS), mostly the cumulants [1-3]. Time coherence was later shown to be a valid separation criterion requiring second-order statistics (SOS) only [4]. More recent research has aimed at taking advantage of prior knowledge about the mixing system or the sources themselves. This may include information on the support of the source of interest, i.e., the time or frequency indices where the desired source is positive or presents significant power [5, 6]. These source

\footnotetext{
* Corresponding author

Email addresses: zarzoso@i3s.unice.fr (V. Zarzoso), ruben@us.es (R. Martín-Clemente), susanah@us.es

(S. Hornillo-Mellado)

${ }^{1}$ Partially supported by the ANR under contract 2010-JCJC-0303-01 "PERSIST".

${ }^{2}$ Partially supported by the "Junta de Andalucía", Spain, under grant P07-TIC-02865.
} 
extraction methods can be formulated by means of conditional SOS, and essentially require the solution of an eigenvalue decomposition (EVD) or generalized EVD problem per iteration.

In the present work, Section 2 puts forward a novel technique of this type based on the first-order statistics of the whitened observations. Originally suggested in [7], the technique is computationally very efficient, which renders it particularly appealing in power-limited scenarios such as embedded systems or wireless sensor networks. The method requires prior knowledge of the positive support of the desired source; in practice, this amounts to the time or frequency snapshot indices where the source of interest is positive. Although the SOS used in [5] are also conditioned on the positive support, the present approach can deal with any source distribution without any modification, and presents lower computational complexity. Moreover, the impact of support estimation inaccuracies on the performance of the new method can be quantified by the theoretical analysis carried out in Section 3. Section 4 analyzes an iterative implementation that may be used when the knowledge of the positive support is uncertain, as may be the case in realistic scenarios where the available prior information is scarce. The experimental study of Section 5 validates the accuracy of the theoretical performance analysis. In the light of these results, some potential applications are discussed in Section 6, while the concluding remarks of Section 7 bring the paper to an end.

\section{Independent Source Extraction Based on the Conditional Mean}

The present work considers the source separation model in instantaneous linear mixtures:

$$
\mathbf{z}(t)=\mathbf{Q s}(t)
$$

where $\mathbf{z}(t) \in \mathbb{R}^{n}$ is the whitened observation vector and $\mathbf{s}(t) \in \mathbb{R}^{n}$ the source vector with components $\mathbf{s}(t)=\left[s_{1}(t), s_{2}(t), \ldots, s_{n}(t)\right]^{\mathrm{T}}$. Matrix $\mathbf{Q} \in \mathbb{R}^{n \times n}$ represents the unitary transformation linking sources and observations after prewhitening. Without loss of generality, we will suppose that $s_{1}(t)$ is the source of interest. The new method essentially relies on two common assumptions in ICA:

A1) The source signals $\mathbf{s}(t)$ are zero mean.

A2) The source signals $\mathbf{s}(t)$ are statistically independent.

The method can be expressed in terms of the conditional mean of the whitened observations, as summarized by the following result.

Proposition 1. The linear extractor

$$
\mathbf{w}=\mathrm{E}\left\{\mathbf{z} \mid s_{1}>0\right\}
$$


recovers the source of interest up to an irrelevant positive scale factor:

$$
y=\mathbf{w}^{\mathrm{T}} \mathbf{z}=\alpha s_{1} \quad \text { with } \alpha \stackrel{\text { def }}{=} \mathrm{E}\left\{s_{1} \mid s_{1}>0\right\}>0 .
$$

Proof. According to model (1) and the linearity of the expectation operator, we have $\mathbf{w}=\mathrm{E}\left\{\mathbf{z} \mid s_{1}>\right.$ $0\}=\mathrm{E}\left\{\mathbf{Q s} \mid s_{1}>0\right\}=\mathbf{Q g}$, where

$$
\mathbf{g}=\mathrm{E}\left\{\mathbf{s} \mid s_{1}>0\right\}
$$

Now, $\mathrm{E}\left\{s_{1} \mid s_{1}>0\right\}=\alpha$ and, by virtue of assumptions A1-A2, $\mathrm{E}\left\{s_{k} \mid s_{1}>0\right\}=\mathrm{E}\left\{s_{k}\right\}=0$, for $1<k \leq n$. Hence, $\mathbf{g}=\alpha \mathbf{e}_{1}$, where $\mathbf{e}_{1}$ denotes the first column of the $(n \times n)$ identity matrix. It follows that $y=\mathbf{w}^{\mathrm{T}} \mathbf{z}=\mathbf{g}^{\mathrm{T}} \mathbf{Q}^{\mathrm{T}} \mathbf{Q} \mathbf{s}=\mathbf{g}^{\mathrm{T}} \mathbf{s}=\alpha s_{1}$.

In practice, applying the above result requires the prior knowledge of indices $t$ where the source of interest presents positive values; in the sequel, this set of indices will be referred to as positive support. The idea behind this source extraction method is simple yet effective: thanks to the independence and zero-mean assumptions A1-A2, averaging the observations over the positive support of the desired source brings out its mixing direction. Under the mixing matrix orthogonality imposed by whitening, the estimated mixing direction can be directly applied on the observed vector to recover the source of interest and cancel out the other sources. The performance of the ICA extractor based on the conditional mean is analyzed in the rest of the paper.

\section{Theoretical Performance Analysis}

This section aims to quantify the source extraction performance obtained by the technique summarized above, including the impact of the imperfect estimation of the desired source positive support. Specifically, we look for a closed-form expression of the theoretical interference-to-signal ratio (ISR) obtained by this source extraction technique. To simplify the following development, we will make two further assumptions:

A3) The source signals are composed of i.i.d. samples.

A4) The source of interest has a symmetric distribution.

Note, however, that the method resulting from Proposition 1 remains valid even if A3-A4 do not hold.

Since we are interested in the practical setting of finite observation lengths, let us assume that the observations are made up of a block of $T$ samples; the corresponding index set is denoted $\mathcal{T}=$ $\{0,1, \ldots, T-1\}$. The set of indices $t \in \mathcal{T}$ for which $s_{1}(t)>0$ and its complement are denoted $\mathcal{T}_{1}$ and 
$\overline{\mathcal{T}}_{1}$, respectively. Let $\mathcal{S}$ represent the index set estimated as the positive support of the source of interest; under assumption $\mathrm{A} 4$, the cardinality of this set is $N \approx T / 2$. The set $\mathcal{S}$ is the union of set $\mathcal{S}_{1}$ composed of $N_{1}$ indices correctly identified, i.e., for which actually $s_{1}(t)>0$, and its complement $\overline{\mathcal{S}}_{1}$ of $\left(N-N_{1}\right)$ indices in $\mathcal{S}$ where $s_{1}(t)<0$; we can express these sets as the intersections $\mathcal{S}_{1}=\mathcal{S} \cap \mathcal{T}_{1}$ and $\overline{\mathcal{S}}_{1}=\mathcal{S} \cap \overline{\mathcal{T}}_{1}$. We denote $r=N_{1} / N$ the correct index classification ratio, that is, the probability that $s_{1}(t)>0$ over the selected indices. The remaining of this section shows how the performance of extractor (2) explicitly depends on this parameter.

Given the observed data block, we first remove the sample mean by subtracting $1 / T \sum_{t \in \mathcal{T}} \mathbf{s}(t)$ from every sample, a customary operation in ICA. Estimator (2) yields the following sample version of the global transformation (4) from the sources to the extractor output:

$$
\hat{\mathrm{g}}=\frac{1}{N} \sum_{t \in \mathcal{S}} \mathbf{s}(t)-\frac{1}{T} \sum_{t \in \mathcal{T}} \mathbf{s}(t)=\frac{1}{T} \sum_{t \in \mathcal{S}} \mathbf{s}(t)-\frac{1}{T} \sum_{t \in \overline{\mathcal{S}}} \mathbf{s}(t)
$$

where $\overline{\mathcal{S}}$ is the complement of $\mathcal{S}$ in $\mathcal{T}$, i.e., $\mathcal{T}=\mathcal{S} \cup \overline{\mathcal{S}}$ with $\mathcal{S} \cap \overline{\mathcal{S}}=\emptyset$, and we have assumed $N=T / 2$. Note that the mean-ergodicity of the sources is necessary for estimating the conditional mean by a sample average. Denoting $\hat{\mathbf{g}}=\left[\hat{g}_{1}, \hat{g}_{2}, \ldots, \hat{g}_{n}\right]^{\mathrm{T}}$, the extractor output can be decomposed as the sum of contributions from the source of interest and the interfering sources:

$$
\hat{y}=\hat{\mathbf{g}}^{\mathrm{T}} \mathbf{s}=\hat{y}_{1}+\hat{b} \quad \text { with } \hat{y}_{1}=\hat{g}_{1} s_{1} \text { and } \hat{b}=\sum_{k=2}^{n} \hat{g}_{k} s_{k} .
$$

To quantify source extraction performance, we define the average ISR per interfering source as:

$$
\mathrm{ISR}=\frac{\mathrm{E}\left\{\hat{b}^{2}\right\}}{(n-1) \mathrm{E}\left\{\hat{y}_{1}^{2}\right\}}=\frac{\sum_{k=2}^{n} \mathrm{E}\left\{\hat{g}_{k}^{2}\right\}}{(n-1) \mathrm{E}\left\{\hat{g}_{1}^{2}\right\}} .
$$

Hence, computing the average ISR requires the calculation of $\mathrm{E}\left\{\hat{g}_{k}^{2}\right\}$, for $1 \leq k \leq n$. To do so, we will resort to the equality $\mathrm{E}\left\{\hat{g}_{k}^{2}\right\}=\mathrm{E}\left\{\hat{g}_{k}\right\}^{2}+\operatorname{var}\left\{\hat{g}_{k}\right\}$. The linearity of the expectation operator and the additivity of the variance under the i.i.d. assumption A3 yield on eqn. (5):

$$
\mathrm{E}\left\{\hat{g}_{k}\right\}=\frac{1}{T} \sum_{t \in \mathcal{S}} \mathrm{E}\left\{s_{k}(t)\right\}-\frac{1}{T} \sum_{t \in \overline{\mathcal{S}}} \mathrm{E}\left\{s_{k}(t)\right\} \quad \operatorname{var}\left\{\hat{g}_{k}\right\}=\frac{1}{T^{2}} \sum_{t \in \mathcal{S}} \operatorname{var}\left\{s_{k}(t)\right\}+\frac{1}{T^{2}} \sum_{t \in \overline{\mathcal{S}}} \operatorname{var}\left\{s_{k}(t)\right\} .
$$

For $k \neq 1$, and taking into account the independence assumption A2 and the unit-power source convention, we have $\mathrm{E}\left\{s_{k}(t)\right\}=0$ and $\operatorname{var}\left\{s_{k}(t)\right\}=1$, for all $t$, so that

$$
\mathrm{E}\left\{\hat{g}_{k}^{2}\right\}=\frac{1}{T} \quad k \neq 1
$$

The computation of $\mathrm{E}\left\{\hat{g}_{1}^{2}\right\}$ is simplified by considering the symmetry assumption A4, which allows us to write:

$$
\mathrm{E}\left\{s_{1}(t)\right\}=\left\{\begin{array}{r}
\alpha, \quad t \in \mathcal{T}_{1} \\
-\alpha, \quad t \in \overline{\mathcal{T}}_{1}
\end{array} \quad \operatorname{var}\left\{s_{1}(t)\right\}=\left(1-\alpha^{2}\right), \quad t \in \mathcal{T}_{1} \text { or } t \in \overline{\mathcal{T}}_{1}\right.
$$


Table 1: Conditional mean $\alpha=\mathrm{E}\{s \mid s>0\}$ for some normalized distributions.

\begin{tabular}{ccccc} 
Bernoulli & Sinusoid & Uniform & Laplacian & Gaussian \\
\hline 1 & $\frac{2 \sqrt{2}}{\pi}$ & $\frac{\sqrt{3}}{2}$ & $\frac{1}{\sqrt{2}}$ & $\sqrt{\frac{2}{\pi}}$
\end{tabular}

where the conditional mean $\alpha$ is defined in eqn. (3). The variance expression has taken into account that, for $t \in \mathcal{T}_{1}, \operatorname{var}\left\{s_{1}(t)\right\}=\operatorname{var}\left\{s_{1} \mid s_{1}>0\right\}=\mathrm{E}\left\{s_{1}^{2} \mid s_{1}>0\right\}-\mathrm{E}\left\{s_{1} \mid s_{1}>0\right\}^{2}=1-\alpha^{2}$ and, by symmetry, $\operatorname{var}\left\{s_{1} \mid s_{1}>0\right\}=\operatorname{var}\left\{s_{1} \mid s_{1}<0\right\}$. Now, $N_{1}$ indices of $\mathcal{S}$ belong to $\mathcal{T}_{1}$ and the remaining $\left(N-N_{1}\right)$ to $\overline{\mathcal{T}}_{1}$; by symmetry, set $\overline{\mathcal{S}}$ contains $\left(N-N_{1}\right)$ indices in $\mathcal{T}_{1}$ and $N_{1}$ in $\overline{\mathcal{T}}_{1}$. Hence, from eqn. (10), the mean and variance in eqn. (8) can be developed for $k=1$ as:

$$
\mathrm{E}\left\{\hat{g}_{1}\right\}=(2 r-1) \alpha \quad \operatorname{var}\left\{\hat{g}_{1}\right\}=\frac{1}{T}\left(1-\alpha^{2}\right) .
$$

The terms in eqn. (11) prove that, for sufficient sample size, $\mathrm{E}\left\{\hat{g}_{1}^{2}\right\}$ is dominated by $\mathrm{E}\left\{\hat{g}_{1}\right\}^{2}$ and, as a result, $\mathrm{E}\left\{\hat{g}_{1}^{2}\right\} \approx \mathrm{E}\left\{\hat{g}_{1}\right\}^{2}=(2 r-1)^{2} \alpha^{2}$. Combining this expression with eqns. (7) and (9), we obtain the theoretical average ISR of the conditional-mean ICA extractor (2):

$$
\mathrm{ISR}=\frac{1}{T(2 r-1)^{2} \alpha^{2}}
$$

This expression explicitly shows the negative impact of inaccuracies in the desired source support estimate, since performance deteriorates as the ratio $r$ of correctly classified indices tends to 0.5 . The symmetry of formula (12) with respect to $r=0.5$ reflects the symmetry of the desired source distribution: estimating its positive support with correct classification ratio $r$ is equivalent to estimating its negative support with ratio $(1-r)$; also, the condition $s_{1}<0$ in eqn. (2) leads to the same source estimate, up to a sign, than the condition $s_{1}>0$. The fitness of this finite-sample approximation and the effect of support estimation errors will be assessed in the experiments of Section 5. Table 1 summarizes the values of the conditional mean $\alpha$ for some common probability distributions.

Remark. If the sample mean is not removed from the observations, eqn. (5) becomes $\hat{\mathbf{g}}=1 / N \sum_{t \in \mathcal{S}} \mathbf{s}(t)$ and eqn. (8) needs to be modified accordingly. The above development then leads to $\operatorname{var}\left\{\hat{g}_{k}\right\}=1 / N=$ $2 / T, k \neq 1$, and $\operatorname{var}\left\{\hat{g}_{1}\right\}=\left(1-\alpha^{2}\right) / N$, whereas the expected values of $\hat{g}_{k}$ remain unchanged, $1 \leq k \leq n$. The asymptotic average ISR will thus experience a $3-\mathrm{dB}$ increase relative to eqn. (12) under the same system conditions. 


\section{Iterative Implementation}

In practical settings the source positive support is unknown or can only be roughly estimated. To overcome this difficulty, we can consider the following iterative implementation:

\section{Algorithm 1}

Step 1. Define the set $\mathcal{S}$ at random or exploiting some available prior knowledge.

Step 2. Estimate the extractor output using the sample version of eqn. (2).

Step 3. From the extractor output (3), obtain a new estimate of $\mathcal{S}$ as $\{t: y(t)>0\}$.

Step 4. Using the new support estimate, go back to Step 2 until convergence.

If required, additional sources may be extracted by using the above algorithm in a deflation scheme, whereby the contribution of each estimated source is subtracted from the observations (e.g., via linear regression) before searching for the next source. For a given sample size and source distribution, eqn. (12) shows that the performance of source extractor (2) depends on parameter $r$, the correct index classification ratio. The support re-estimation from the extractor output in Step 3 will generally produce a new value of this parameter. In turn, application of Step 2 on the new support estimate will yield a new ISR according to (12), and so forth. Hence, the theoretical evolution of the above iterative algorithm can be analyzed by computing the probability of correct support estimation, defined as $r^{\prime}=P\left(s_{1}>0 \mid \hat{y}>0\right)$, achieved by Steps 2-3.

To this end, first note that Bayes theorem $P\left(s_{1}>0 \mid \hat{y}>0\right)=P\left(s_{1}>0\right) P\left(\hat{y}>0 \mid s_{1}>0\right) / P(\hat{y}>0)$ reduces to $P\left(\hat{y}>0 \mid s_{1}>0\right)$ since $P\left(s_{1}>0\right)=P(\hat{y}>0)=0.5$ for symmetric distributions. Working out $P\left(\hat{y}>0 \mid s_{1}>0\right)$ requires the integration of $p_{\hat{y} \mid s_{1}>0}(u)$ over $[0,+\infty)$, where $p_{\hat{y} \mid s_{1}>0}(u)$ is the probability density function (pdf) of $\hat{y}$ given $s_{1}>0$. To compute this pdf, we note that the terms due to the source of interest and the interference in eqn. (6) are independent by virtue of assumption A2. Also, recall that the mean of $\hat{g}_{1}$ dominates its variance for sufficient sample size [eqn. (11)], so that the desired source term in eqn. (6) can be approximated by $\hat{y}_{1} \approx \mathrm{E}\left\{\hat{g}_{1}\right\} s_{1}=\hat{\alpha} s_{1}$, with

$$
\hat{\alpha} \stackrel{\text { def }}{=}(2 r-1) \alpha
$$

The pdf of the extractor output over the positive support of the desired source is then: $p_{\hat{y} \mid s_{1}>0}(u)=$ $\int_{-\infty}^{+\infty} p_{\hat{\alpha} s_{1} \mid s_{1}>0}(\tau) p_{\hat{b}}(u-\tau) \mathrm{d} \tau=2 \int_{0}^{+\infty} p_{\hat{\alpha} s_{1}}(\tau) p_{\hat{b}}(u-\tau) \mathrm{d} \tau$, where the second equality follows from $p_{\hat{\alpha} s_{1} \mid s_{1}>0}(\tau)=$ $0, \tau<0$, and $p_{\hat{\alpha} s_{1} \mid s_{1}>0}(\tau)=2 p_{\hat{\alpha} s_{1}}(\tau), \tau>0$, for symmetric distributions. From the Central Limit Theorem, the interference term $\hat{b}$ behaves as a zero-mean Gaussian random variable with variance

$$
\sigma^{2}=\sum_{k=2}^{n} \mathrm{E}\left\{\hat{g}_{k}^{2}\right\}=\frac{n-1}{T}
$$


where we have made use of eqn. (9). All the above considerations yield:

$$
r^{\prime}=P\left(s_{1}>0 \mid \hat{y}>0\right)=\int_{0}^{+\infty} p_{\hat{y} \mid s_{1}>0}(u) \mathrm{d} u=2 \int_{0}^{+\infty} \int_{0}^{+\infty} p_{\hat{\alpha} s_{1}}(\tau) \frac{1}{\sqrt{2 \pi} \sigma} \exp \left(-\frac{(u-\tau)^{2}}{2 \sigma^{2}}\right) \mathrm{d} \tau \mathrm{d} u .
$$

The change of variable $v=(u-\tau) /(\sqrt{2} \sigma)$ and the error function $\operatorname{erf}(x)=\frac{2}{\sqrt{\pi}} \int_{0}^{x} e^{-v^{2}} \mathrm{~d} v$ finally lead to:

$$
r^{\prime}=\frac{1}{2}+\int_{0}^{+\infty} p_{\hat{\alpha} s_{1}}(\tau) \operatorname{erf}\left(\frac{\tau}{\sqrt{2} \sigma}\right) \mathrm{d} \tau
$$

The integral term on the right-hand side of eqn. (15) quantifies how the correct support estimation probability will depart on average from 0.5 , which is the worst-case scenario. If $\sigma$ is small enough then $\operatorname{erf}\left(\tau / \sqrt{2} \sigma^{-1}\right) \approx 1$ for $\tau>0$, and the above integral yields $r^{\prime} \approx 1$ as soon as $\hat{\alpha} \neq 0$, i.e., $r \neq 0.5$ [eqn. (13)]. According to eqn. (14), a sufficient sample length guarantees a small value of $\sigma$.

As an example, let us consider the case of a desired source with symmetric Bernoulli distribution, in which $p_{\hat{\alpha} s_{1}}(\tau)=\frac{1}{2} \delta(\tau-\hat{\alpha})$ for $\tau>0$, and $\hat{\alpha}=(2 r-1)$ [eqn. (13) and Table 1]. Plugging these terms and expression (14) into eqn. (15) leads to the fixed-point like update:

$$
\left(2 r^{\prime}-1\right)=\operatorname{erf}\left(\sqrt{\frac{T}{2(n-1)}}(2 r-1)\right) .
$$

If positive constant $\gamma$ is large enough, then $\operatorname{erf}(\gamma x)>x$ for $x \in[0,1)$ and $\operatorname{erf}(\gamma) \approx 1$. Therefore, for sufficient sample size the above iteration will converge to a fixed point near $r^{\prime}=1$ if the initial value of $r$ is greater than 0.5 .

Before closing this section, remark that the conditional-mean ICA extractor update in Step 2 requires just $n$ products per iteration, regardless of the data block size $T$; these are the $n$ multiplications by $1 / T$ in eqn. (5). This contrasts with the $O\left(n^{2} T\right)$ products of the conditional SOS techniques of $[5,6]$, or even the $O(n T)$ products of FastICA [3], one of the most computationally attractive ICA methods proposed to date.

\section{Experimental Results}

A few numerical experiments first assess the accuracy of the single-iteration theoretical analysis carried out in Section 3 as well as the impact of support estimation errors. This is followed by the empirical analysis of the iterative implementation developed in the previous section.

Single iteration. T-sample blocks of unitary mixtures of $n$ independent sources are observed, mimicking the whitened-sensor model of eqn. (1). In the first experiment, the positive support of the source of interest is assumed to be perfectly known. Figure 1 (left) shows the ISR obtained by a single iteration of the conditional-mean ICA extractor (2) for different distributions of $n=20$ sources. Results are averaged 

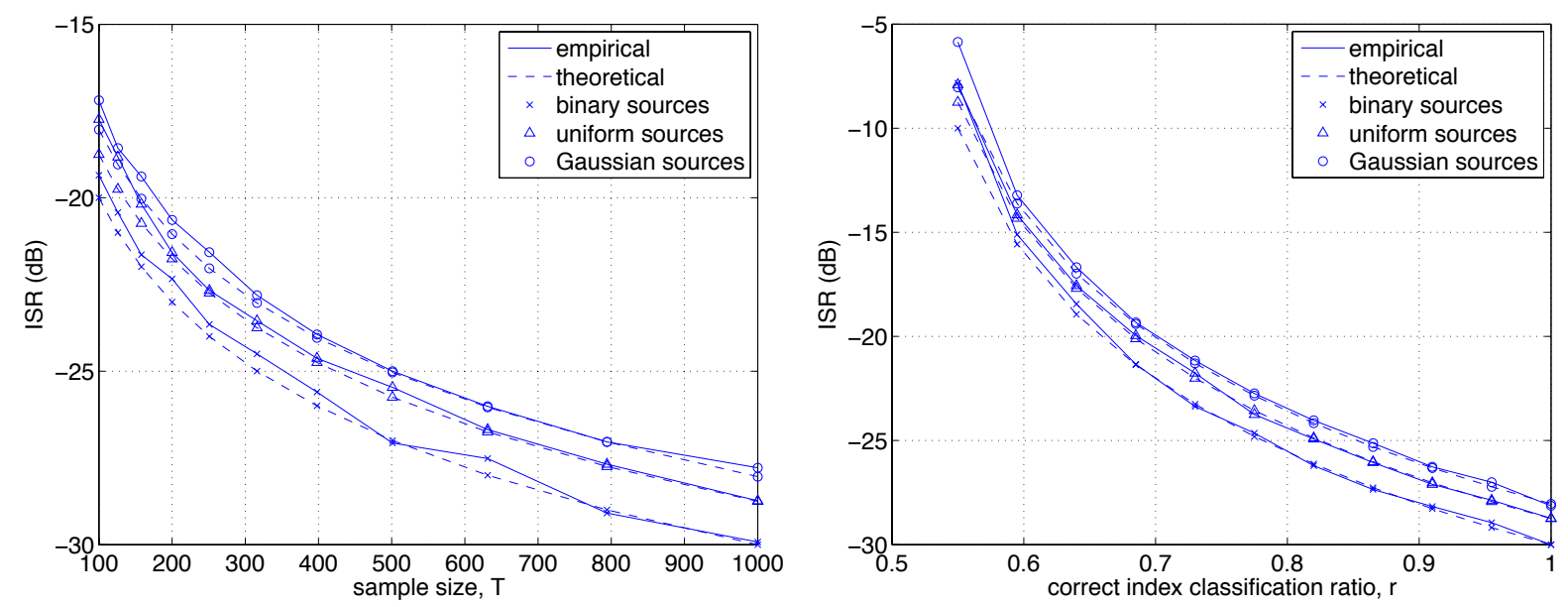

Figure 1: Performance of a single iteration of the conditional-mean ICA extractor. (Left) ISR versus sample size for mixtures of $n=20$ sources and $r=1$. (Right) ISR versus correct index classification ratio for mixtures of $n=20$ sources and $T=1000$ samples. In both plots, the ISR is averaged over 100 Monte Carlo runs.

over 100 independent Monte Carlo runs. The fitness of approximation (12) with the $\alpha$ values of Table 1 is very precise in all cases, even for low sample size. Next, we fix $T=1000$ samples and repeat the experiment for different values of the correct index classification ratio $r$. Figure 1 (right) shows that performance deteriorates as $r \rightarrow 0.5$, as predicted by eqn. (12). Again, this asymptotic approximation proves very accurate in all cases.

Iterative implementation. The iterative implementation of Section 4 is applied on unitary mixtures of $n=10$ symmetric binary sources composed of $T=1000$ samples. Figure 2 shows the average trajectories of $r$ and ISR obtained by Algorithm 1 over 100 Monte Carlo runs, for different initial values of the correct index classification ratio, labelled as $r_{1}$ in the plots. Also shown are the theoretical trajectories defined by (16) and the corresponding ISR values given in (12). For the considered mixture setting, the predicted $r$-value evolution (Fig. 2, left) is quite accurate if the initial value of $r$ is not too close to 0.5. The expected ISR trajectory (Fig. 2, right) is less precise as $r_{1}$ decreases, but can at least match the kurtosis-based FastICA [3] while recovering the source of interest. Considering the computational load per iteration, the quality-cost trade-off superiority of the conditional-mean ICA is even more apparent, despite FastICA's theoretical global cubic convergence.

\section{Discussion}

Successfully targeting specific sources with the conditional-mean ICA technique analyzed in this paper requires some prior knowledge of their positive support. In digital communications, support information 

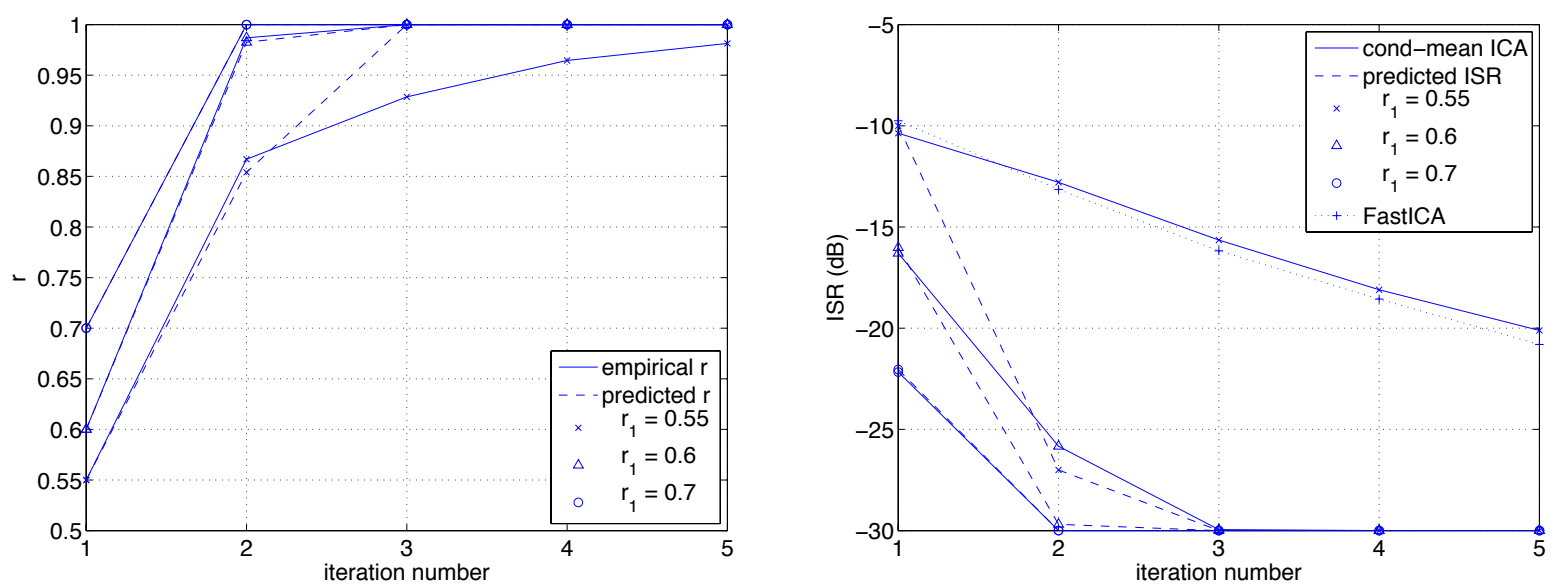

Figure 2: Performance of the iterative implementation of the conditional-mean ICA extractor for mixtures of $n=10$ binary sources, $T=1000$ samples and 100 Monte Carlo runs. (Left) Trajectories of the correct index classification ratio for different initial values of this parameter. (Right) ISR trajectories for different initial values of the correct index classification ratio. FastICA's ISR values are computed without reference to any particular source.

would be available over the pilot sequence, so that the proposed method could be combined with classical training-based receivers yielding semi-blind equalizers with reduced complexity. A number of space-time coded systems like Alamouti's scheme lead to observation models with a unitary mixing matrix [8], thus sparing the need for prewhitening and preventing its asympotic performance limitations [2]. The proposed technique would be fully based on first-order statistics in such scenarios.

The signal of interest is partially known in certain signal processing problems involving the electrocardiogram (ECG). During atrial fibrillation, the most common sustained cardiac arrhythmia, the atrial activity signal to be analyzed in the ECG is masked by the ventricular activations or heartbeats, but remains visible in the intervals between them [9]. The absence of interference in such intervals would increase the accuracy of the initial support selection for extracting the atrial sources over the whole ECG recording using the conditional-mean ICA technique.

In some cases, applying the proposed method to particular real-world problems may call for applicationdependent modifications, which, due to lack of space, are out of the scope of this letter.

\section{Conclusions}

This work has presented a novel method for ICA based on the conditional first-order statistics of the observed data. Compared to ICA methods based on SOS or HOS, the new method presents a very low computational complexity, which makes it particularly convenient in power-limited scenarios such as 
wireless sensor networks or embedded systems. The method's extraction performance depends on the accurate estimation of the positive support of the source of interest, but further iterations can improve the precision of the initial support estimate and thus the source extraction quality for sufficient sample size. Future work should analyze the source extraction capabilities of the proposed method in fully blind scenarios, explore its extension to complex-valued data, and evaluate its comparative performance in real-world applications.

\section{References}

[1] P. Comon, Independent component analysis, a new concept?, Signal Processing 36 (3) (1994) 287-314, Special Issue on Higher-Order Statistics.

[2] J.-F. Cardoso, B. H. Laheld, Equivariant adaptive source separation, IEEE Transactions on Signal Processing 44 (12) (1996) 3017-3030.

[3] A. Hyvärinen, E. Oja, A fast fixed-point algorithm for independent component analysis, Neural Computation 9 (7) (1997) 1483-1492.

[4] A. Belouchrani, K. Abed-Meraim, J.-F. Cardoso, E. Moulines, A blind source separation technique using second-order statistics, IEEE Transactions on Signal Processing 45 (2) (1997) 434-444.

[5] B. Xerri, B. Borloz, An iterative method using conditional second-order statistics applied to the blind source separation problem, IEEE Transactions on Signal Processing 52 (2) (2004) 313-328.

[6] R. Phlypo, V. Zarzoso, I. Lemahieu, Source extraction by maximizing the variance in the conditional distribution tails, IEEE Transactions on Signal Processing 58 (1) (2010) 305-316.

[7] R. Martín-Clemente, S. Hornillo-Mellado, J. L. Camargo-Olivares, Fast independent component analysis using a new property, in: Proc. IWANN-2011, International Work Conference on Artificial Neural Networks, Torremolinos, Spain, 2011, pp. 477-483.

[8] H. J. Pérez-Iglesias, J. A. García-Naya, A. Dapena, L. Castedo, V. Zarzoso, Blind channel identification in Alamouti coded systems: a comparative study of eigendecomposition methods in indoor transmissions at 2.4 GHz, European Transactions on Telecommunications 19 (7) (2008) 751-759.

[9] V. Zarzoso, Extraction of ECG characteristics using source separation techniques: exploiting statistical independence and beyond, in: A. Naït-Ali (Ed.), Advanced Biosignal Processing, Springer, Berlin, 2009, Ch. 2, pp. 15-47. 